\title{
Self-management practices among type 2 diabetes patients attending primary health-care centres in Medina, Saudi Arabia
}

\author{
K.A. Al Johani, ${ }^{1}$ G.E. Kendall ${ }^{2}$ and P.D. Snider ${ }^{2}$
}

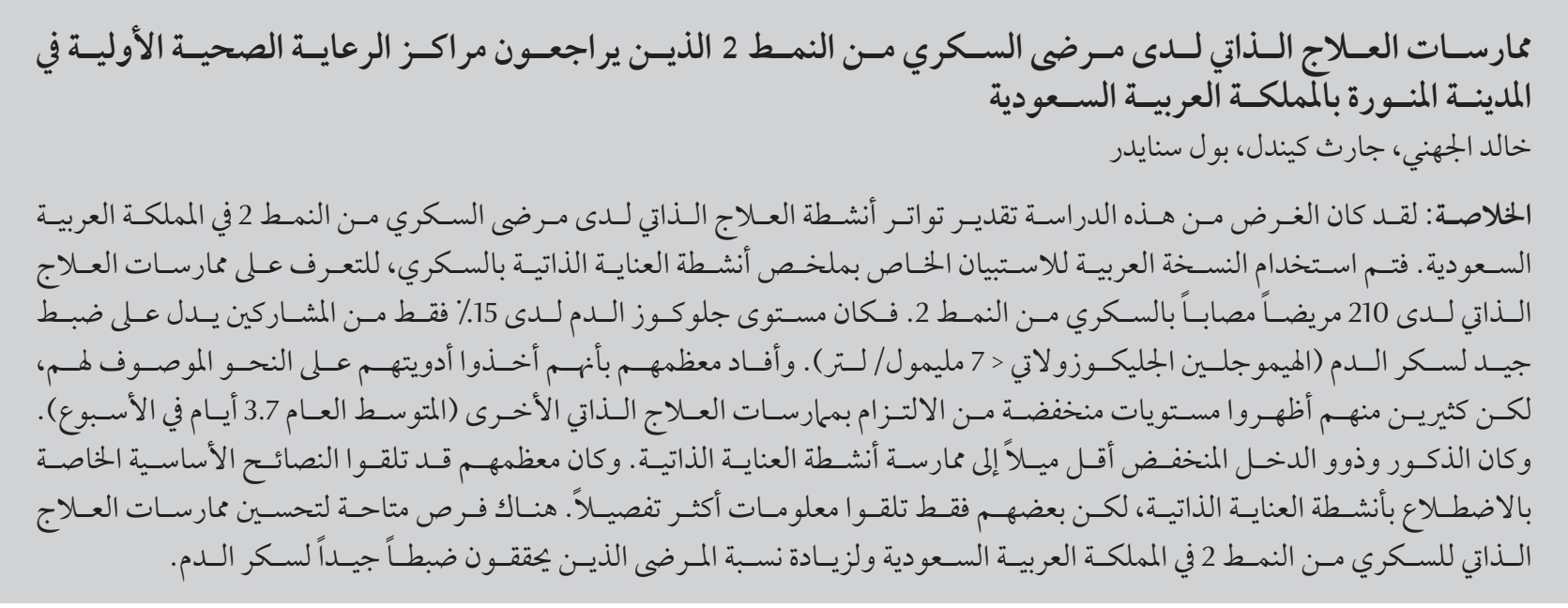

ABSTRACT The purpose of this study was to estimate the frequency of self-management activities among people who have type 2 diabetes in Saudi Arabia. The Arabic version of the Summary of Diabetes Self-care Activities questionnaire was used to identify self-management practices among 210 patients with type 2 diabetes mellitus. Only 15\% of participants had a blood glucose level indicative of good glycaemic control (glycosylated haemoglobin $\leq 7 \mathrm{mmol} / \mathrm{L}$ ). Most reported that they took their medication as prescribed, but many demonstrated low levels of compliance with other self-management practices (overall mean 3.7 days per week). Males and those with lower incomes were less likely to practise self-care activities. Most were given basic advice to undertake self-care activities, but only some were given more detailed information. There are opportunities to improve type 2 diabetes mellitus self-management practices in Saudi Arabia and increase the proportion of patients who achieve good glycaemic control.

Pratiques d'auto-prise en charge chez des patients atteints de diabète de type $\mathbf{2}$ fréquentant des centres de soins de santé primaires à Médine (Arabie saoudite)

RÉSUMÉ L'objectif de la présente étude était d'estimer la fréquence des activités d'auto-prise en charge chez des personnes atteintes de diabète de type 2 en Arabie saoudite. La version en langue arabe du questionnaire Summary of Diabetes Self-care Activities a été utilisée pour identifier les pratiques d'auto-prise en charge chez 210 patients atteints de diabète sucré de type 2 . Seuls $15 \%$ des participants présentaient un taux glycémique révélateur d'un bon contrôle de leur glycémie (hémoglobine glycosylée $\leq 7 \mathrm{mmol} / \mathrm{L}$ ). La plupart indiquaient avoir pris leur traitement comme prescrit, mais ils étaient nombreux à présenter des taux d'observance faibles pour d'autres pratiques d'auto-prise en charge (moyenne d'observance globale hebdomadaire : 3,7 jours sur sept). Les hommes et les personnes ayant des revenus plus faibles étaient moins susceptibles de pratiquer des activités d'autosoins. Pour la majorité, ils avaient reçu des conseils élémentaires pour réaliser des activités d'autosoins, mais seule une minorité d'entre eux avait eu des informations plus détaillées. Des opportunités existent pour améliorer les pratiques d'auto-prise en charge du diabète de type 2 en Arabie saoudite et augmenter le pourcentage de patients qui parviennent à un bon contrôle du taux de glycémie.

${ }^{7}$ Ministry of Health, Medina, Saudi Arabia (Correspondence to K.A. Al Johani: aljohani.khalid@gmail.com). ${ }^{2}$ Curtin University of Technology, School of Nursing and Midwifery, Perth, Western Australia, Australia.

Received: 24/12/14; accepted: 28/05/15 


\section{Introduction}

In the Eastern Mediterranean Region, $7.7 \%$ of the population has been diagnosed with diabetes, a figure which has been estimated to double in the next 20 years (1). In Saudi Arabia, research provides some evidence that diabetes is not well managed (2-4). Poor access to health care, ineffective management of chronic disease and poor referral patterns were identified as major challenges to optimum diabetes health care in the country (5-7).

Clearly, a great deal more research is required about the management of diabetes in Saudi Arabia. The present study follows from the successful translation of the Summary of Diabetes Self-care Activities (SDSCA) instrument into the Arabic language and its validation using a Saudi population sample $(8,9)$. This is the first estimation of the frequency of self-management activities in Saudi Arabia that is based on international criteria, as reflected in the study instrument's subscales. The study may inform future projects to improve diabetes care and encourage researchers to undertake similar studies on a wider population base.

\section{Methods}

\section{Setting and sample}

This paper was part of a larger study that was conducted by June 2011 . Out of 35 health-care centres in the Medina catchment area (population 1100093 ), the study was conducted in 3 out of 4 main health centres (average population covered by each centre was about 18 000) (10). The study locations were representative of Saudi Arabia's diabetes chronic disease management programmes (5). All type 2 diabetes patients attending the clinic during the 2 -week data collection period were invited through posters and approached by the researcher and the researcher assistant to participate in the study (approximately 600). A convenience sample of 210 people ( 70 from each centre with males and females represented equally) agreed to participate in the study.

The inclusion criteria were: diagnosed with type 2 diabetes; able to participate without a mental disability that could affect his/her decisions; no physical disability affecting self-care; and above 26 years of age. Patients who were not registered at the chronic diseases clinics at the study locations were excluded.

\section{Procedure}

Participants were recruited through posters that were located in the study locations. In addition, the researcher and the researcher assistant invited patients in the waiting room to participate. Information about the study was mainly presented verbally in detail. Participants were informed that they may record their responses directly on the questionnaire or they may delegate a research assistant or nurse to the task. Although some participants recorded their responses themselves, the majority asked a research assistant or nurse to do so.

For each participant data about the glycosylated haemoglobin ( $\mathrm{HbAlC}$ ) level or fasting blood glucose (FBG) level that had been measured in the past 3 months were obtained from the medical records. The recruitment process was implemented until the required participant quota $(n=70)$ was attained in each centre, with a mean duration of 2 weeks for each study location.

Ethical approval was obtained from the authors' affiliated medical and research institutions in both Saudi Arabia and Australia. Verbal consent was obtained from the participants before commencing data collection.

\section{Instruments}

The study questionnaire consisted of 4 main sections: sociodemographic data; time since the diagnosis with diabetes and any complications experienced; the Arabic version of the SDSCA (ASDSCA); and the extended A-SDSCA. In addition, a blood sample was drawn to obtain a recent $\mathrm{HbAlC}$ or FBG level.

\section{Sociodemographic variables}

Sociodemographic data were re-categorized to allow statistical comparisons between groups. Age was categorized as $<65$ and $\geq 65$ years. Income was categorized as a binary variable based on annual income in Saudi riyals (SR) (> 50000 versus $\leq 50000$ ). Low-income group in this study were those who with annual income up to SR 50 000, taking into account the assumption that they were renting their homes.

\section{Time since first diagnosis and inci- dence of complications}

Previous studies have indicated that there is a positive relationship between diabetes duration and the incidence of medical complications (11). Duration of diabetes incidence was later collapsed and presented as a binary variable with categories $<8$ years and $\geq 8$ years. Participants were also asked to state if they had microvascular and macrovascular complications, such as cardiac, eye and kidney health problems (1).

\section{A-SDSCA questionnaire}

The main section of the A-SDSCA instrument consists of 4 self-care subscales: diet (2 items), exercise ( 2 items), blood glucose testing (2 items) and foot care (2 items) and 2 questions each about medications and smoking. The first 4 subscales ask the respondents to record on how many days (from 0 to 7 ), they have performed the specified selfcare activity. Detailed information was presented in an earlier paper (9). Respondents are also asked if they smoked. If they answered "yes," they were asked how many cigarettes or waterpipes they smoked per day.

\section{A-SDSCA extended questionnaire}

Items included in the extension to the A-SDSCA aim to identify the amount of self-management diabetes education 
the participants have received. The extension consists of 4 sections: diet ( 8 items), exercise (6 items), blood glucose testing ( 5 items) and medications (5 items). To complete these sections, respondents are required to tick boxes for the specific advice that they have received from a doctor, nurse, dietician or diabetes educator at their health-care centre.

\section{Blood glucose level}

HbAlC level taken from the participant's medical record was the measure of blood glucose used if the test had been undertaken during the last 3 months. However, due to data limitation in the study locations, FBG was recorded as an alternative. Participants were identified as having a controlled blood glucose level if they had $\mathrm{HbAlC}$ $\leq 7 \mathrm{mmol} / \mathrm{L}$ or FBG $\leq 130 \mathrm{mg} / \mathrm{dL}$ (12). Any value above these thresholds was considered to be an uncontrolled blood glucose level.

\section{Data analysis}

Data analysis was undertaken using SPSS, version 17 software. The main A-SDSCA instrument was scored by calculating the mean for each item then calculating the mean for each subscale and computing the mean of the total A-SDSCA scale. Binary cut-off variables were also derived to identify the proportion of participants whose self-care management was within recommended self-management practices $(13,14)$. Items from the extension part of the instrument were scored by computing frequencies and percentages. A series of binary variables were derived to summarize data regarding the sociodemographic characteristics of participants, length of time since diagnosis, diabetes complications and blood glucose level.

The frequencies of variables were tabulated. Next, a number of independent samples $t$-tests were undertaken to determine if any of the self-management activities undertaken by participants varied according to their sociodemographic characteristics, time since diagnosis, presence of complications and blood glucose levels. Statistical significance was defined at the $P<0.05$ level (2-tailed). Finally, standard linear regression was performed to assess the independent relationship between the explanatory variables and the total ASDSCA score and to determine the proportion of variance explained by these variables.

\section{Results}

\section{Descriptive analysis}

\section{Participants' characteristics}

As Table 1 shows, the majority of participants in this sample ( $82 \%)$ were aged 26-65 years and there was an equal number of males and females. One-third of participants (33\%) had no formal education, and almost 55\% had an annual income of SR $<50000$ (US\$
$<13$ 000). Two-thirds (66\%) of the recruited sample had been diagnosed with type 2 diabetes mellitus for more than 8 years. Blood glucose testing records indicated that only $15 \%$ of the participants had a blood glucose level in the range recommended by the 2008 American Diabetes Association (ADA) guideline. Medical complications had already affected some participants, in the form of heart disease (4\%), hypertension (30\%), kidney disease (2\%) and eye disease (17\%).

\section{Mean scores for A-SDSCA scales and binary outcomes}

The mean scores for A-SDSCA scales and binary outcomes for A-SDSCA scales are presented in Table 2. This shows that participants demonstrated low to medium mean levels of selfmanagement; however, standard deviations of around 2.0 or more indicated a considerable variation in the number of days per week these activities were undertaken.

\begin{tabular}{|c|c|c|}
\hline \multirow[t]{2}{*}{ Variable } & \multicolumn{2}{|c|}{ Respondents } \\
\hline & No. & $\%$ \\
\hline \multicolumn{3}{|l|}{ Age (years) } \\
\hline $26-65$ & 172 & 82 \\
\hline$>65$ & 37 & 18 \\
\hline \multicolumn{3}{|l|}{ Sex } \\
\hline Male & 105 & 50 \\
\hline Female & 105 & 50 \\
\hline \multicolumn{3}{|c|}{ Income/year(Saudi riyals) } \\
\hline$<50000$ & 116 & 55 \\
\hline$\geq 50000$ & 94 & 45 \\
\hline \multicolumn{3}{|l|}{ Education level } \\
\hline No formal education & 70 & 33 \\
\hline Formal educated & 139 & 67 \\
\hline \multicolumn{3}{|l|}{ Disease duration (years) } \\
\hline$<8$ & 71 & 34 \\
\hline$\geq 8$ & 138 & 66 \\
\hline \multicolumn{3}{|l|}{ Blood glucose } \\
\hline Controlled & 30 & 15 \\
\hline Uncontrolled & 174 & 85 \\
\hline
\end{tabular}

Data were missing in some categories. 
Binary cut-off scores, which identify the proportion of participants whose self-care management was within ADA recommended guidelines, are also presented in Table 2. According to the information provided, $29 \%$ of participants were unable to manage their diet, $47 \%$ did not take enough exercise, $85 \%$ did not measure their blood glucose frequently, $41 \%$ were lax in their foot care and $25 \%$ did not take their medication as prescribed. A total of 27 participants (13\%) were smokers at the time of data collection.

\section{Responses to A-SDSCA extension questions}

Table 3 presents responses to the ASDSCA extension questions and shows that $91 \%$ of the participants indicated they were advised to follow a low-fat diet plan by their health-care providers; however, 5 patients (2\%) reported they did not receive any dietary recommendations. The majority of participants (87\%) were advised to engage in low-level exercise, such as walking on a daily basis, while 7 participants (3\%) reported they did not receive advice that stressed the importance of exercise in everyday self-management practices. Other recommendations are presented in detail in Table 3 .

\section{Bivariate analysis}

Bivariate relationships between patients' mean self-care scores and their sociodemographic/clinical characteristics are reported for the 5 self-care subscales of the A-SDSCA (diet, exercise, blood glucose, foot care and medication) in Table 4. It can be seen that there was a significant relationship between age and foot care, with those aged $\geq 65$ years spending less time on average practising foot-care activities than younger participants.

Table 4 also shows that there were significant relationships between sex and diet, blood glucose testing and foot care, with males on average spending less time practising these activities (diffs $-1.03, P<0.001 ;-0.775, P<0.01$; and $-2.63, P<0.001$ respectively).
Formal education was not significantly associated with most self-care activities. However, it can be seen that those with formal education spent less time practising foot care (diff -1.02; $P$ $<0.05$ ). Income was another potential risk factor and was significantly associated with blood glucose testing. Those with lower incomes spent less time on this activity (diff $-1.16 ; P<0.001$ ).

Duration of time with diabetes was associated with good exercise self-management, but not with other activities. Participants who had been diagnosed for $\geq 8$ years spent significantly fewer days per week exercising than those diagnosed for $<8$ years (diff 0.806 ; $P$ $<0.05$ ). Those with controlled blood glucose spent more days on good diet than those with uncontrolled blood glucose (diff 1.07; $P<0.001$ ) and more often tested their blood glucose (diff $0.886 ; P<0.05)$.

Finally, Table 4 shows that participants who smoked spent more time than those who did not on diet and foot care (diff 1.29; $P<0.001$ and diff 1.59;

\begin{tabular}{|c|c|c|c|c|c|c|c|}
\hline \multirow[t]{3}{*}{ Subscale/item } & \multicolumn{3}{|c|}{ Binary outcomes } & \multirow{2}{*}{\multicolumn{2}{|c|}{$\begin{array}{l}\text { Item scores } \\
\text { (days/week })\end{array}$}} & \multirow{2}{*}{\multicolumn{2}{|c|}{$\begin{array}{l}\text { Subscale scores } \\
\text { (days/week })\end{array}$}} \\
\hline & \multirow{2}{*}{$\begin{array}{c}\text { No. of } \\
\text { days/week } \\
\text { activity } \\
\text { performed }^{\text {a }}\end{array}$} & \multicolumn{2}{|c|}{ Respondents } & & & & \\
\hline & & No. & $\%$ & Mean & SD & Mean & SD \\
\hline Diet & & & & & & 3.60 & 1.70 \\
\hline Q1. Follow a healthful eating plan & $\leq 2$ & 61 & 29 & 3.48 & 2.13 & - & - \\
\hline Q2. Follow eating plan over the past month & $\geq 3$ & 149 & 71 & 3.58 & 2.14 & - & - \\
\hline Exercise & & & & & & 3.02 & 2.17 \\
\hline Q3. Participate in at least 30 min exercise & $\leq 2$ & 98 & 47 & 3.34 & 2.33 & - & - \\
\hline Q4. Participate in specific exercise session & $\geq 3$ & 109 & 52 & 2.63 & 2.32 & - & - \\
\hline Blood sugar checks & & & & & & 2.24 & 1.90 \\
\hline Q5. Test your blood sugar & $\leq 4$ & 179 & 85 & 2.43 & 2.04 & - & - \\
\hline Q6. Test your blood sugar as recommended & $\geq 5$ & 28 & 13 & 2.02 & 1.88 & - & - \\
\hline Foot care & & & & & & 3.49 & 2.37 \\
\hline Q7. Check your feet & $\leq 2$ & 87 & 41 & 3.72 & 2.60 & - & - \\
\hline Q8. Inspect the inside of your shoes & $\geq 3$ & 118 & 56 & 3.34 & 2.66 & - & - \\
\hline Medication & $\leq 6$ & 52 & 25 & & & 6.26 & 0.59 \\
\hline Overall & - & - & - & - & - & 3.72 & 1.15 \\
\hline
\end{tabular}

${ }^{a}$ Based on the American Diabetes Association 2008 guideline; ${ }^{b}$ Scale range 0-7.

$S D=$ standard deviation. 


\begin{tabular}{|c|c|c|c|c|}
\hline \multirow[t]{2}{*}{ Which of the following has your health-care team advised you to do? } & \multicolumn{2}{|c|}{ Yes } & \multicolumn{2}{|c|}{ No } \\
\hline & No. & $\%$ & No. & $\%$ \\
\hline Follow a low-fat eating plan & 189 & 91 & 19 & 9 \\
\hline Follow a complex carbohydrate diet & 154 & 73 & 55 & 27 \\
\hline Reduce calories you eat to lose weight & 138 & 66 & 71 & 34 \\
\hline Eat lots of food high in dietary fibre & 150 & 71 & 59 & 29 \\
\hline Received advice about my diet & 204 & 98 & 5 & 2 \\
\hline Get low level exercise (such as walking) on a daily basis & 182 & 87 & 27 & 13 \\
\hline Exercise for at least 20 min at least 3 times a week & 81 & 39 & 128 & 61 \\
\hline Fit exercise into your daily routine & 89 & 43 & 120 & 57 \\
\hline Received advice about exercise & 202 & 97 & 7 & 3 \\
\hline Test your blood sugar using a machine to read the results & 199 & 95 & 9 & 5 \\
\hline Test your urine for sugar & 91 & 44 & 11 & 56 \\
\hline Received advice about blood sugar testing & 201 & 97 & 7 & 3 \\
\hline
\end{tabular}

$P<0.001$ ), and they were more regular in taking their medication (diff 1.16; $P$ $<0.05)$.

\section{Multivariate analysis}

The results of linear regression analysis, the independent relationships between the various characteristics of the participants and their total self-management scores, are presented in Table 5. Particie pants' age, sex, income, level of education, length of time since diagnosis, level of glucose control and smoking habit accounted for $25 \%$ of the variability in their total self-management score $\left(R^{2}=\right.$ 0.251 ). Table 5 shows that women were much more likely than men toundertake appropriate diabetes self-management $(\beta=0.321 ; P<0.001)$ and smokers were more likely than non-smokers not to do so $(\beta=-0.192 ; P<0.01)$.

Other statistically significant results were found for income and the level of glucose control. Participants with an income SR > 50000 were more likely than those with a lower income to undertake appropriate diabetes care activities $(\beta=0.129 ; P=0.055)$. Those with a controlled glucose level were more likely than those with uncontrolled glucose to undertake appropriate diabetes care activities $(\beta=-0.122 ; P=0.054)$. Participant's age, educational level and time since diagnosis had little impact on total self-management scores after adjustment for all other characteristics.

\section{Discussion}

\section{Self-care management practices}

A major finding of the study was that only $15 \%$ of participants had an $\mathrm{HbAlC}$ level $\leq 7 \mathrm{mmol} / \mathrm{L}$ (i.e. indicative of good glycaemic control) (12). This result is very similar to previous research findings in Saudi Arabia. Azab found that $21 \%$ of patients had a FBG < 4-6 mmol/L (2) and Al-Hussein showed that $21 \%$ of the sample had an acceptable $\mathrm{HbA1C}$ level of $<7$ $\mathrm{mmol} / \mathrm{L}(4)$.

Another important finding was that participants generally had poor glycaemic control, even though most of them reported that they took their

\begin{tabular}{|c|c|c|c|c|c|}
\hline \multirow[t]{2}{*}{ Variable (groups) } & \multicolumn{5}{|c|}{ t-test for differences in mean scores on self-care subscales } \\
\hline & Diet & Exercise & $\begin{array}{l}\text { Blood sugar } \\
\text { checks }\end{array}$ & Foot care & Medication \\
\hline Age $(<65$ yrs $=1, \geq 65$ yrs $=2)$ & 0.61 & $1.86^{\mathrm{b}}$ & -0.34 & $2.81^{\mathrm{b}}$ & -0.58 \\
\hline Sex $($ male $=1$, female $=2)$ & -4.60 & $-1.30^{\mathrm{a}, \mathrm{b}}$ & $-2.90^{\mathrm{a}, \mathrm{b}}$ & $-9.49^{b}$ & -0.13 \\
\hline Formal education $(\mathrm{no}=1$, yes $=2$ ) & 0.93 & $-1.31^{b}$ & $-0.67^{b}$ & $2.22^{\mathrm{b}}$ & 0.22 \\
\hline Income $(\leq$ SR $50000=1$, > SR $50000=2)$ & $-1.50^{\mathrm{a}, \mathrm{b}}$ & $-0.95^{\mathrm{a}, \mathrm{b}}$ & $-4.61^{b}$ & $1.56^{\mathrm{b}}$ & -0.61 \\
\hline Disease duration $(<8$ yrs $=1, \geq 8$ yrs $=2)$ & $0.37^{b}$ & $2.57^{\mathrm{b}}$ & 0.16 & $1.78^{\mathrm{b}}$ & $-1.40^{\mathrm{b}}$ \\
\hline Blood glucose $($ controlled $=1$, uncontrolled $=2$ ) & $2.23^{b}$ & $0.91^{\mathrm{b}}$ & $2.30^{\mathrm{b}}$ & $1.32^{\mathrm{b}}$ & $-1.08^{\mathrm{b}}$ \\
\hline Smoking status (yes $=1$, no $=2$ ) & $3.81^{\mathrm{b}}$ & $1.33^{b}$ & 0.59 & $3.28^{b}$ & $2.65^{\mathrm{b}}$ \\
\hline
\end{tabular}

${ }^{a}$ Equal variance not assumed; ${ }^{b} P \leq 0.05$. 


$\begin{aligned} & \text { Table } 5 \text { Multivariate relationships between sociodemographic/clinical variables } \\
& \text { of the type 2 diabetes patients and their overall self-care scores }\end{aligned}$
\begin{tabular}{lcccc}
\hline Predictors & B & SE B & $\boldsymbol{\beta}$ & P-value \\
Age $\geq 65$ years & -0.256 & 0.222 & -0.082 & 0.251 \\
Female sex & 0.753 & 0.173 & 0.321 & 0.000 \\
Income > SR 50 000 & 0.305 & 0.158 & 0.129 & 0.055 \\
Formal education & -0.067 & 0.181 & -0.027 & 0.712 \\
Disease duration $\geq 8$ years & -0.128 & 0.161 & -0.052 & 0.429 \\
Glucose uncontrolled & -0.403 & 0.208 & -0.122 & 0.054 \\
Smoker & -0.684 & 0.237 & -0.192 & 0.004 \\
\hline
\end{tabular}

$F=9.30, P=0001, R^{2}=0.251$.

medication as prescribed. The finding that the study participants demonstrated low levels of compliance with most other self-management practices indicates that they did not understand the importance of these practices for moderating their blood glucose levels and minimizing the possibility of developing undesirable complications of the disease. Furthermore, the finding that only $15 \%$ of participants had good glycaemic control is strong evidence that medication alone is not the answer to the effective management of type 2 diabetes mellitus. These findings reflect serious limitations in the way type 2 diabetes mellitus selfmanagement is promoted in the study locations.

With regard to self-care management, while $71 \%$ of participants reported eating a healthy diet 3 or more days per week, only $12 \%$ reported adhering to an optimal diet 7 days per week. This indicates how hard it is to maintain a healthy diet in Saudi Arabia, where social connections are strong and people are expected to attend all social gatherings to which they are invited to eat the traditional food provided (15). This is similar to the situation in the Philippines, where the people have a strong connection with rice as a source of carbohydrate (16). In the Arab context, there is a high probability that traditional social and cultural practices work against the efforts made by people with type 2 diabetes mellitus to maintain an optimal diet.
As to exercise, only $53 \%$ of the sample reported that they exercised 3 or more days per week as recommended. This percentage is lower than that reported in similar Asian studies. In a recent Chinese study, it was reported that $40 \%$ of participants who had type 2 diabetes mellitus maintained healthy exercise practices throughout the week (17). In another study, $54 \%$ of Korean people with type 2 diabetes mellitus who visited a general practitioner maintained good exercise practices (11). A possible reason for the large proportion of participants in the current study not exercising is that $18 \%$ were over 65 years of age. They may not be able to perform regular exercise due to general physical decline more likely to be attributable to the hot climate in Saudi Arabia, which constrains simple exercises such as walking. Also, Saudi Arabian culture is quite unlike Asian culture in that it does not encourage other types of healthy activities for older people such as yoga and tai chi (18).

The overall mean numbers of days per week that self-care activities were practised in this study was 3.7. While the practice of self-care activities is lower than that reported by studies in the United States that have also used the SDSCA instrument $(14,16)$, it is comparable to that found in a recent study in Jordan, where the culture and context are similar to Saudi Arabia (13). In our study, participants' levels of or poor health. However, it is much self-management practices were based on whether or not they were performing within classification thresholds that were adapted from the $\mathrm{ADA}$ guidelines of 2008 (12). Whereas the results of this study showed that $29 \%$ of Saudi participants did not follow their recommended diet 3 or more days per week, the Jordan study found that approximately $19 \%$ of the Jordanian sample did not do so (13). Similarly, $68 \%$ of participants did not achieve the recommended physical exercise threshold ( $\leq$ 2 days/week) compared with $47 \%$ in the current study. Conversely, it was reported that $38 \%$ of the Jordanian participants tested their blood glucose at home at least 5 days per week, whereas only $15 \%$ of the Saudi participants did so. Compliance with medication was reasonable in both studies, but higher in Jordan ( $91 \%$ versus $75 \%$ ). These results concur with other studies conducted in developed countries that have found a general preference for people to take medication for any health problem, as it is easier than changing their own behaviour $(19,20)$.

\section{Explanatory variables}

Given the heterogeneity of sociodemographic characteristics among people with type 2 diabetes mellitus that has been noted in all countries, it is important to know if there are certain groups within the population who are likely to attend to self-care activities more or less than others. The results of this study show that in Saudi Arabia those who were older, male or low-income earners were less likely to practise specific self-care activities. While older age was solely associated with foot care, and low income was solely associated with blood glucose testing, male sex was significantly related to diet, blood glucose testing and foot care.

The results also showed that those who had good glycaemic control were more likely to manage their diet 
appropriately and test their blood glucose more regularly; those who had no formal education were more likely to care for their feet; and those who smoked were more likely to attend to diet, foot care and medication. In contrast to more developed countries, people in Saudi Arabia who have little formal education and thus low social status are more likely, rather than less likely, to follow medical advice. As to smoking, the 27 smokers in the study were predominantly younger people. They might not be involved in social gatherings to the same extent as older people, and thus not subjected to the unhealthy diet of rice and meat typically offered at these events. Another possible explanation is that smoking has decreased their appetite for food (21).

With regard to the practice of self-care activities, sex, income, glycaemic control and smoking were all independent explanatory factors. Given that glycaemic control is most likely an outcome of good self-management rather than a predictor of it and that smoking is known to do much more harm than good, it follows then that sex and income remain as the 2 potential issues for health-care providers to consider. Worldwide, low income has been identified as a barrier to attaining recommended self-management practices such as blood glucose monitoring (22). Low income restricts patients' ability to access health-care services and purchase specialized equipment. Low income, or financial strain, also acts as a proxy measure for the experience of life stress, which is associated with increased morbidity and mortality (23). The fact that health-care services are free in Saudi Arabia does not mean people who are on a low income have equal access or opportunity. As mentioned previously, the health-care system does not, for example, provide a glucometer for all patients with type 2 diabetes mellitus. Furthermore, it is likely that those on low incomes are not able to visit a health-care centre regularly due to difficulties with work scheduling, child-care and transport.

A possible explanation for better dietary practices among women could be that they are more likely to be mindful of their diet than men are (24). Because women are considered a dependent population group in Saudi, it is not surprising that they more readily adhere to advice about diabetes self-management (18). The culture, social norms and religious commands encourage those of lower status to abide by those in important educational and religious positions.

\section{Advice given by health-care providers}

The responses to the A-SDSCA extension questions in the current study make it clear that a great majority of participants were given at least basic advice by their health-care providers to undertake self-care activities. However, it is also evident that fewer people were given more detailed information about diet and exercise, such as eating fruit and vegetables and exercising continuously for at least 20 minutes 3 times per week. In this regard, the advice given fell short of meeting international standards of self-management education (12). The failure to encourage patients to practise self-management activities may be considered a major limitation of the current health education approach in primary health care in Saudi Arabia. Further research is required in Saudi to substantiate this claim or to refute it. If it is substantiated, the health-care authorities must act to improve the standard of self-management education given to people with type 2 diabetes mellitus.

\section{Limitations of the study}

The study has a number of strengths, but there are also 2 potential limitations. First, as participants were purposefully sampled to achieve equal representation of the sexes, and two-thirds of those people who were invited to participate declined to do so, sample bias is possible. However, the characteristics of those who did participate were very similar to those obtained in other Saudi studies and there is little indication that they were a socioeconomically advantaged group $(4,6,25)$. More importantly, there was no indication that people declined to participate because they were illiterate. In fact, many people requested that the questions be read to them and that the researcher complete the questionnaire. Second, the exploratory cross-sectional study design did not allow cause-and-effect relationships between explanatory variables and self-management outcomes to be examined.

\section{Conclusion}

There are opportunities to improve type 2 diabetes mellitus self-management practices in Saudi Arabia. The contribution of nurses and other team members is scant in the literature. Appropriately qualified diabetes educators could take the lead in supporting patients to play an active role in managing their treatment plan, exploring effective selfmanagement strategies, and learning to take advantage of the Saudi Ministry of Health and community resources. Due to the small sample in the current study, further studies are strongly recommended.

\section{Acknowledgements}

The authors gratefully thank the research participants for time and commitment to complete the study. Pursuing this study was inapplicable without the tremendous support of the chronic diseases nurses and physicians at the Department of Primary Health Care in Medina, Saudi Arabia.

Funding: None.

Competing interests: None declared. 


\section{References}

1. About diabetes [Internet]. Brussels: International Diabetes Federation; 2015; (http://www.idf.org/about-diabetes, accessed 3 July 2015).

2. Azab AS. Glycemic control among diabetic patients. Saudi Med J. 2001 May;22(5):407-9. PMID:11376381

3. Abdelmoneim I, Al-Homrany MA. Health education in the management of diabetes at the primary health care level: is there a gender difference? East Mediterr Health J. 2002 Jan;8(1):18-23. PMID:15330556

4. Al-Hussein FA. Diabetes control in a primary care setting: a retrospective study of 651 patients. Ann Saudi Med. 2008 JulAug;28(4):267-71. PMID:18596404

5. Al-Ahmadi H, Roland M. Quality of primary health care in Saudi Arabia: a comprehensive review. Int J Qual Health Care. 2005 Aug;17(4):331-46. PMID:15883128

6. Al-Khaldi YM, Khan MY. Audit of a diabetic health education program at a large Primary Health Care Center in Asir region. Saudi Med J. 2000 Sep;21(9):838-42. PMID:11376360

7. Uddin I, Ahmad TJ, Kurkuman ARA, Iftikhar R. Diabetes education: its effects on glycemic control. Ann Saudi Med. 2001 Jan-Mar;21(1-2):120-2. PMID:17264611

8. Toobert DJ, Hampson SE, glasgow RE. The summary of diabetes self-care activities measure: Results from 7 studies and a revised scale. Diabetes Care. 2000 Jul;23(7):943-50. PMID:10895844

9. AlJohani KA, Kendall GE, Snider PD. Psychometric Evaluation of the Summary of Diabetes Self-Care Activities-Arabic (SDSCA-Arabic): Translation and Analysis Process. J Transcult Nurs. 2014 May 19 PMID:24841470

10. General census of population and housing 2010. Riyadh: Ministry of Economy and Planning; 2010.

11. Lee H, Ahn S, Kim Y. Self-care, self-efficacy, and glycemic control of koreans with diabetes mellitus. Asian Nurs Res (Korean Soc Nurs Sci). 2009 Sep;3(3):139-46. PMID:25030472

12. American Diabetes Association.Standards of medical care in diabetes-2008. Diabetes Care. 2008 Jan;31 Suppl 1:S12-54. PMID:18165335

13. Khattab M, Khader YS, Al-Khawaldeh A, Ajlouni K. Factors associated with poor glycemic control among patients with type 2 diabetes. J Diabetes Complications. 2010 Mar-Apr;24(2):84-9. PMID:19282203

14. Katon W, Russo J, Lin EH, Heckbert SR, Ciechanowski P, Ludman EJ, et al. Depression and diabetes: factors associated with major depression at five-year follow-up. Psychosomatics. 2009 Nov-Dec;50(6):570-9. PMID:19996227
15. AIQuaiz AM, Tayel SA. Barriers to a healthy lifestyle among patients attending primary care clinics at a university hospital in Riyadh. Ann Saudi Med. 2009 Jan-Feb;29(1):30-5. PMID:19139617

16. Jordan DN, Jordan JL. Self-care behaviors of Filipino-American adults with type 2 diabetes mellitus. J Diabetes Complications. 2010 Jul-Aug;24(4):250-8. PMID:19615920

17. Xu Y, Pan W, Liu H. Self-management practices of Chinese Americans with type 2 diabetes. Nurs Health Sci. 2010 Jun;12(2):228-34. PMID:20602696

18. Musaiger S. Women and educational leadership in a Muslim society. In: Sobehart H, editor. Women leading education across the continents: Sharing the spirit, fanning the flame. Lanham (MD): Rowman and Littlefield Education; 2009. pp. $128-42$.

19. Hayes RP, Bowman L, Monahan PO, Marrero DG, McHorney CA. Understanding diabetes medications from the perspective of patients with type 2 diabetes: prerequisite to medication concordance. Diabetes Educ. 2006 May-Jun;32(3):404-14. PMID:16772656

20. Murphy M, Saunders J, Campbell M, Jackson B, Berlowitz D. The process of implementing the Stanford Model Chronic Disease Self-Management Program: the Northern Hospital experience. Aust J Prim Health. 2003;9(2-3):127-31.

21. Mineur YS, Abizaid A, Rao Y, Salas R, DiLeone RJ, Gündisch $D$, et al. Nicotine decreases food intake through activation of POMC neurons. Science. 2011 Jun 10;332(6035):1330-2. PMID:21659607

22. Levine DA, Allison JJ, Cherrington A, Richman J, Scarinci IC, Houston TK. Disparities in self-monitoring of blood glucose among low-income ethnic minority populations with diabetes, United States. Ethn Dis. 2009 Spring;19(2):97-103. PMID:19537217

23. Commission on Social Determinants of Health. Closing the gap in a generation: health equity through action on the social determinants of health. Geneva: World Health Organization; 2008 (http://whqlibdoc.who.int/publications/2008/9789241563703_eng.pdf, accessed 3 July 2015).

24. Anschutz DJ, Van Strien T, Engels RC. Exposure to slim images in mass media: television commercials as reminders of restriction in restrained eaters. Health Psychol. 2008 Jul;27(4):401-8. PMID:18642997

25. Albaz R. [Poverty line in Saudi Arabia]. Al Watan. 12 May 2005 [in Arabic]. 